\title{
La lectura como actividad de ocio en escolares asturianos de $6^{\circ}$ de Primaria
}

\author{
María del Rosario NEIRA PIÑEIRO \\ Universidad de Oviedo \\ Facultad de Formación del Profesorado y Educación \\ Departamento de Ciencias de la Educación \\ neiramaria@uniovi.es
}

Recibido: 19 de febrero de 2015

Aceptado: 3 de marzo de 2015

\section{RESUMEN}

Este trabajo analiza los resultados de una investigación sobre hábitos lectores del alumnado asturiano de $6^{\circ}$ de Educación Primaria en su tiempo libre. El estudio utiliza una muestra de 324 escolares de centros públicos urbanos, con edades comprendidas entre los 11 y 12 años, para analizar datos relativos a la actitud hacia la lectura, la importancia de ésta entre las actividades de ocio y la frecuencia lectora.

Más de la mitad de los escolares manifiesta una actitud bastante favorable hacia la lectura, y ésta es una de sus actividades de ocio habituales, aunque dedican más tiempo a ver televisión, practicar deportes, usar el ordenador y escuchar música. La mayoría son lectores frecuentes, aunque los no lectores y lectores ocasionales ascienden hasta un 12,96\%. Los escolares encuestados leen principalmente libros, seguidos de revistas y cómics, mientras que poco más de un tercio dice leer periódicos frecuentemente.

Esta investigación revela también algunas variaciones en actitud hacia la lectura y frecuencia lectora según el centro educativo de procedencia que se pueden relacionar con variables de tipo socio-económico. Además, se observan diferencias entre niños y niñas, pues éstas superan a sus compañeros varones en interés y frecuencia lectora, además de manifestar también divergencias en las preferencias de materiales de lectura.

Palabras clave: hábitos lectores, lectura, actividades de ocio, Educación Primaria

\section{Reading as leisure activity in Asturian $6^{\text {th }}$ grade elementary school children}

\begin{abstract}
This paper analyses the results obtained by a research about 6th grade elementary school children's reading habits in their free time. The study uses a sample of 324 Asturian children, aged 11 to 12 , from urban state schools. This allows to analyse the attitude towards reading, the relevance of reading among other leisure activities and reading frequency.

Most of schoolchildren show a quite positive attitude towards reading, which is one of their usual activities in their free time, although they spend more time watching TV, playing sports, using computers and listening music. Most children are frequent readers, but the non-
\end{abstract}


readers and occasional readers amount to $12.96 \%$. The surveyed children read mainly books, followed by magazines and comics, and a few more than a third read journals frequently.

This research also reveals some variations in attitude towards reading and reading frequency between the pupils depending on the schools, which can be related to social and economic variables. Moreover, there are relevant differences between boys and girls, as girls surpass their male classmates in interest and reading frequency, and show some differences in the reading materials that they prefer.

Key words: reading habits, reading, leisure activities, primary education

\section{La lecture comme activité de loisir chez les élèves asturiens de $6^{\text {ème }}$ année à l'école primaire}

\section{RÉSUMÉ}

Ce travail analyse les résultats d'une recherche sur les habitudes de lecture des élèves asturiens de 6ème année à l'école primaire, pendant leur temps libre. La recherche, auprès d'un échantillon de 324 enfants d'écoles publiques, de 11 à 12 ans, analyse des données concernant l'attitude vers la lecture, son importance comme activité de loisir et la fréquence de lecture.

Plus de la moitié des élèves manifeste une attitude favorable envers la lecture, celle-ci étant une de leurs activités de loisir préférées, si bien ils consacrent plus de temps à voir la télévision, pratiquer des sports, utiliser l'ordinateur et écouter de la musique. La plupart lit fréquemment, mais les non-lecteurs et les lecteurs occasionnels sont, au total, 12,96\%.

Les sujets enquêtés lisent principalement des livres, suivis de magazines et bandes dessinées, tandis qu'environ un tiers des enfants lit fréquemment des journaux. Cette recherche révèle aussi quelques variations concernant l'attitude envers la lecture et la fréquence de lecture selon l'école, ce qui peut être lié à des variables de type socio-économique. En plus, on observe des différences entre les garçons et les filles, car celles-ci surpassent les garçons en ce qui concerne leur intérêt et fréquence de lecture. En outre, les enfants manifestent des différentes selon leur sexe dans le type de matériaux qu'ils préfèrent lire.

Mots-clés: habitudes de lecture, lecture, activités de loisir, école primaire

SUMARIO: 1. Introducción; 2. Diseño de la investigación y metodología; 2.1. Objetivos; 2.2. Método; 2.3. Muestra; 3. Exposición y discusión de los resultados; 3.1. Valoración de la lectura; 3.2. Razones para leer; 3.3. Causas del rechazo a la lectura; 3.4. La lectura como actividad de ocio; 3.5. Frecuencia de actividades de ocio; 3.6. Frecuencia de lectura; 3.7. Lectura en soportes digitales: uso del ordenador en el tiempo libre; 3.8. Frecuencia de lectura según tipos de textos; 4. Conclusiones; 5. Prospectiva; 6. Bibliografía.

\section{INTRODUCCIÓN}

Actualmente, la lectura en general y el desarrollo de hábitos lectores en los niños y adolescentes son objeto de especial interés tanto para los investigadores en didáctica de la lengua y literatura como para las instituciones educativas. El desarrollo 
del hábito lector es contemplado en la legislación educativa actual ${ }^{1}$ no solamente como uno de los objetivos del área de Lengua Castellana y Literatura sino también como un objetivo general para toda la etapa de Educación Primaria. Asimismo, la LOMCE señala, al igual que la LOE, la obligatoriedad de destinar un tiempo diario a la lectura en esta etapa educativa, con el propósito de contribuir a desarrollar hábitos lectores en el alumnado.

La valoración positiva de la lectura, así como su práctica habitual, aparecen recogidas en el currículo actual junto al desarrollo de la competencia lectora, pudiendo ser considerados todos ellos como aspectos estrechamente interrelacionados. El desarrollo de la comprensión lectora y la animación a la lectura se complementan, pues la capacidad de leer comprensivamente es un requisito para interesarse por la lectura y, a la inversa, las prácticas de animación lectora inciden positivamente en la mejora de la comprensión (Mata, 2011, 133-134). Los estudios realizados ponen de manifiesto cómo los mejores resultados en competencia lectora según PISA son parejos al grado de interés y compromiso hacia la lectura, que no sólo supone leer mucho, sino también leer textos de diverso tipo (Livannakylä y Välijärvi, 2006, 230). Del mismo modo, los informes sobre las pruebas PISA revelan cómo los estudiantes que disfrutan leyendo poseen una competencia lectora claramente superior a los que no leen por placer (OCDE, 2010, 30-31). En la misma dirección, otras investigaciones muestran que la existencia de una actitud positiva hacia el hecho de leer, unida al disfrute lector, junto con la práctica regular de la lectura fuera de la escuela, están estrechamente relacionadas con la mejora del rendimiento en lectura (Clark, Woodley y Lewis, 2011, 5 y 7). Por otra parte, la práctica de la lectura no sólo incide en el desarrollo de la competencia lectora en general, sino que es también una actividad central para la formación literaria (Dueñas, 2013, 136).

Esta preocupación por crear una actitud favorable hacia los libros y por consolidar hábitos de lectura se refleja en las reflexiones teóricas y en las propuestas prácticas desarrolladas en torno al concepto de "animación lectora". A pesar de que la asiduidad es un elemento esencial del hábito lector, más importante aún es la buena disposición del lector, el deseo de leer y la capacidad para obtener placer o provecho en el acto de la lectura (Lomas y Matas, 2007, 11-12).

En este aspecto, las investigaciones ponen de manifiesto la complejidad del concepto de "hábito lector", dado que no solamente se define por la cantidad de obras leídas sino también otras variables, como el tipo de textos leídos, el gusto por la lectura, etc. (Valdés, 2013). Así, según Mireia Manresa, la persona que posee "un buen hábito lector adquirido" es aquélla que lee habitualmente una cantidad elevada de textos de características diversas (en cuanto a género, temática, complejidad, etc.) y al mismo tiempo "es capaz de valorar lo que lee" (Manresa, 2009, 44-45).

${ }^{1}$ Este aspecto, presente en la Ley Orgánica de Educación (LOE), se mantiene en La Ley Orgánica para la Mejora de la Calidad Educativa (LOMCE). 
Aunque la creación de hábitos lectores depende de numerosos condicionantes y debería ser tarea de toda la sociedad (Lomas y Matas, 2007, 13), suele recaer principalmente en dos ámbitos: la familia y la escuela. Diversas investigaciones señalan que el entorno familiar es determinante (Mata, 2011, 160-ss; Clark y Hawkins, 2010): la existencia de modelos lectores adultos, el nivel de estudios de los progenitores, la práctica de la lectura en familia, etc. inciden positivamente en la formación del hábito lector. También se destaca la importancia que posee la escuela (Lomas y Mata, 2007, 13), y, por consiguiente, la responsabilidad de los profesores en la formación de lectores. Aunque la consolidación de hábitos lectores más allá del ámbito escolar es un asunto individual, los docentes tienen la responsabilidad de facilitar "experiencias positivas de lectura en el periodo escolar" (Dueñas, 2013, 142) así como lograr que sus alumnos desarrollen el nivel de competencia lectora necesario para que puedan llegar a ser lectores habituales (Moreno, 2005, 155).

En este aspecto, es esencial la tarea orientadora del docente en la selección de textos para leer, lo que requiere conocer previamente los gustos e intereses de los estudiantes en materia de lecturas (Polanco, 2007, 38). Por ello, resulta fundamental la obtención de datos sobre la importancia concedida a la lectura en el tiempo libre, la actitud de los escolares hacia esta actividad, así como sus hábitos lectores en relación con diversos tipos de textos.

Esta preocupación por los hábitos lectores, no sólo de los más jóvenes sino de la población en general, se refleja en numerosos estudios realizados tanto en España como en otros países. Las investigaciones cuantitativas sobre este tema se inician en España en 1964 (Cerrillo, Larrañaga y Yubero, 2002, 71-ss). A partir de esta fecha se suceden diversas encuestas realizadas tanto a nivel nacional como autonómico ${ }^{2}$, así como otros estudios tanto generales como referidos a algún aspecto o a un determinado sector de la población (Cerrillo y García Padrino, 1996; Gómez Soto, 1999; Gasol y Arànega, 2000, 27-30; Moreno Sánchez, 2001; Cerrillo, Larrañaga y Yubero, 2002, 61-ss; Millán, 2002; Murillo et al., 2003; Larrañaga y Yubero, 2005; Reynolds, 2005; Cerrillo, 2007, 101; Muñoz y Hernández, 2008; Colomer, 2009; Gil Flores, 2009; Manresa Potroni, 2009). En el ámbito internacional existen diversos trabajos sobre el mismo tema, algunos de ellos orientados específicamente a conocer los hábitos de los lectores más jóvenes, como los realizados por el NCRCL, el National Literacy Trust en Inglaterra, o el Centro Australiano de Literatura Infantil y Juvenil, entre otros (Reynolds, 2005, 87-ss).

Según las investigaciones realizadas, las variables más importantes en relación con los hábitos lectores son el nivel de estudios, la edad, el sexo y el tamaño del

\footnotetext{
${ }^{2}$ Un listado de las encuestas realizadas en el ámbito nacional se puede consultar en la obra indicada de Cerrillo, Larrañaga y Yubero (2002). Muchos de estos informes, así como otros realizados en diferentes países, se recogen en la página web del Observatorio de la Lectura y el Libro: http://www.mcu.es/libro/MC/ObservatorioLect/informes.html
} 
hábitat. En términos generales, leen más las mujeres con estudios superiores y residentes en poblaciones con mayor número de habitantes, además de detectarse una mayor frecuencia lectora en determinadas franjas de edad (Conecta, 2011, 48-ss). En lo referido a los más jóvenes, se observan algunas tendencias generales, como el alto índice de lectores infantiles frecuentes, la influencia determinante de la familia, la mayor frecuencia de lectura entre estudiantes de sexo femenino o la preferencia por los libros frente a otro tipo de materiales escritos.

Muchos de los estudios citados se centran en los lectores adultos, la población en general, o en sectores relativamente amplios de la población infantil y adolescente. Ahora bien, nuestra investigación se ciñe a una franja de edad muy específica (11 a 12 años), que coincide con el fin de una etapa educativa y supone un antes y un después en la evolución de los hábitos lectores: éstos aumentan progresivamente durante la infancia hasta los 11 años aproximadamente, momento en el cual inician un descenso que continúa durante la adolescencia, siendo más acusado entre los chicos (Muñoz y Hernández, 2008, 609). La franja de edad elegida se corresponde con un momento de especial relevancia en lo que a hábitos lectores se refiere: por un lado, podemos constatar el resultado de la evolución lectora de los estudiantes y de la paulatina conformación de sus gustos e intereses a lo largo de la Educación Primaria. Por otro, se apuntan tendencias nuevas que se prolongarán durante la Educación Secundaria Obligatoria, como la pérdida o consolidación de lectores según los casos o la transformación de las preferencias lectoras de los estudiantes. Nuestro propósito es extraer datos que ayuden a comprender las tendencias que caracterizan los hábitos lectores de los escolares en un momento decisivo como es el último ciclo de la Educación Primaria. En el análisis de los datos observaremos en qué medida los hábitos lectores de los escolares de 11 a 12 años de edad coinciden con los datos más genéricos referidos a niños y adolescentes en general, haciendo referencia a aquellas diferencias o coincidencias más significativas ${ }^{3}$. Por último, utilizaremos la información obtenida para proponer algunas líneas o pautas de actuación para la promoción de la lectura y la educación lectora en general desde el ámbito educativo.

\section{DISEÑO DE LA INVESTIGACIÓN Y METODOLOGÍA}

\subsection{Objetivos}

El principal objetivo era recabar información sobre los hábitos lectores de los escolares de $6^{\circ}$ de Primaria de centros públicos de Oviedo. El análisis se centró en la lectura voluntaria realizada fuera de la escuela, ya que es la que revela la actitud real de los niños hacia los libros.

\footnotetext{
${ }^{3}$ Utilizaremos como término de comparación los últimos barómetros de lectura realizados por Conecta \& Consulting por encargo de la Federación de Gremios de Editores de España, fundamentalmente el correspondiente al año 2011.
} 
A través de esta investigación se obtuvieron datos cuantitativos y cualitativos relativos a diferentes aspectos que inciden en el hábito lector. De todas las cuestiones analizadas, se recogen y comentan aquí las relativas a la valoración de la lectura, el lugar que ésta ocupa como actividad de ocio de los escolares, la frecuencia lectora en general y la frecuencia lectora según tipos de textos.

\subsection{Método}

Se utilizó un cuestionario escrito de 25 ítems ${ }^{4}$, con preguntas de respuesta abierta y cerrada, de opción única o múltiple según los casos. Dicho cuestionario se aplicó, durante el curso 2010/2011, en diferentes colegios públicos de la localidad de Oviedo, capital del Principado de Asturias, perteneciente a un municipio con un total de 225.005 habitantes $^{5}$, ubicado en la zona metropolitana central de Asturias.

\subsection{Muestra}

La investigación se llevó a cabo con una muestra de 324 escolares de $6^{\circ}$ de Primaria (168 niños y 156 niñas), con edades comprendidas entre los 11 y 12 años, procedentes de 8 colegios públicos de la ciudad de Oviedo. En el curso académico correspondiente, el número total de estudiantes de $6^{\circ}$ de Primaria matriculados en centros públicos de Oviedo ascendía a 868, por lo que la muestra seleccionada constituye un $37,33 \%$ del universo.

Para que dicha muestra fuera representativa, se seleccionaron centros educativos ubicados en diferentes barrios, tanto céntricos como periféricos, con diferentes características poblacionales. Se incluyeron algunos próximos al centro, emplazados en zonas urbanas con una población de clase media. En el otro extremo, y pasando por centros con situaciones intermedias, se seleccionaron también colegios ubicados en áreas periurbanas o en el extrarradio, con población de clase media-baja y una mayor presencia de inmigrantes.

El 15,43\% de los encuestados había nacido fuera de España, con un claro predominio de escolares procedentes de países de habla española (68\% de los inmigrantes encuestados). Dado que no se han detectado diferencias significativas entre éstos y los nacidos en España, no se hará referencia a esta variable en el análisis de los datos.

La mayoría de los encuestados procedían de familias en las que trabajan ambos progenitores. Casi todas las familias trabajaban en el sector terciario y, en menor medida, en el secundario. La mayoría de los padres y madres tenían trabajos que no exigen titulación superior, siendo más habituales los relacionados con el comercio, la industria, la construcción, la hostelería, el transporte, los servicios domésticos y

\footnotetext{
${ }^{4}$ Los datos comentados en este artículo se corresponden con los ítems 1 a 5 del cuestionario.

${ }^{5}$ Población del municipio de Oviedo según los censos de población y viviendas de 2011 (datos del INE)
} 
el sector servicios en general. Sin embargo, en algunos de los colegios sí se ha constatado un porcentaje más elevado de padres y madres con titulaciones superiores, así como la presencia de profesiones vinculadas a la enseñanza, los servicios sanitarios y la administración pública.

\section{EXPOSICIÓN Y DISCUSIÓN DE LOS RESULTADOS}

\subsection{Valoración de la lectura}

En primer lugar, se intentó averiguar la valoración que los encuestados hacían de la lectura por placer (figura 1). Algo más de la mitad (54,01\%) manifiesta una actitud bastante favorable hacia esta actividad, pues declara que le gusta leer mucho o bastante. Un porcentaje significativo $(33,72 \%)$ revela un interés más bajo hacia esta actividad, que solamente le gusta "algo", mientras que un número más reducido $(12,34 \%)$ la rechaza claramente.

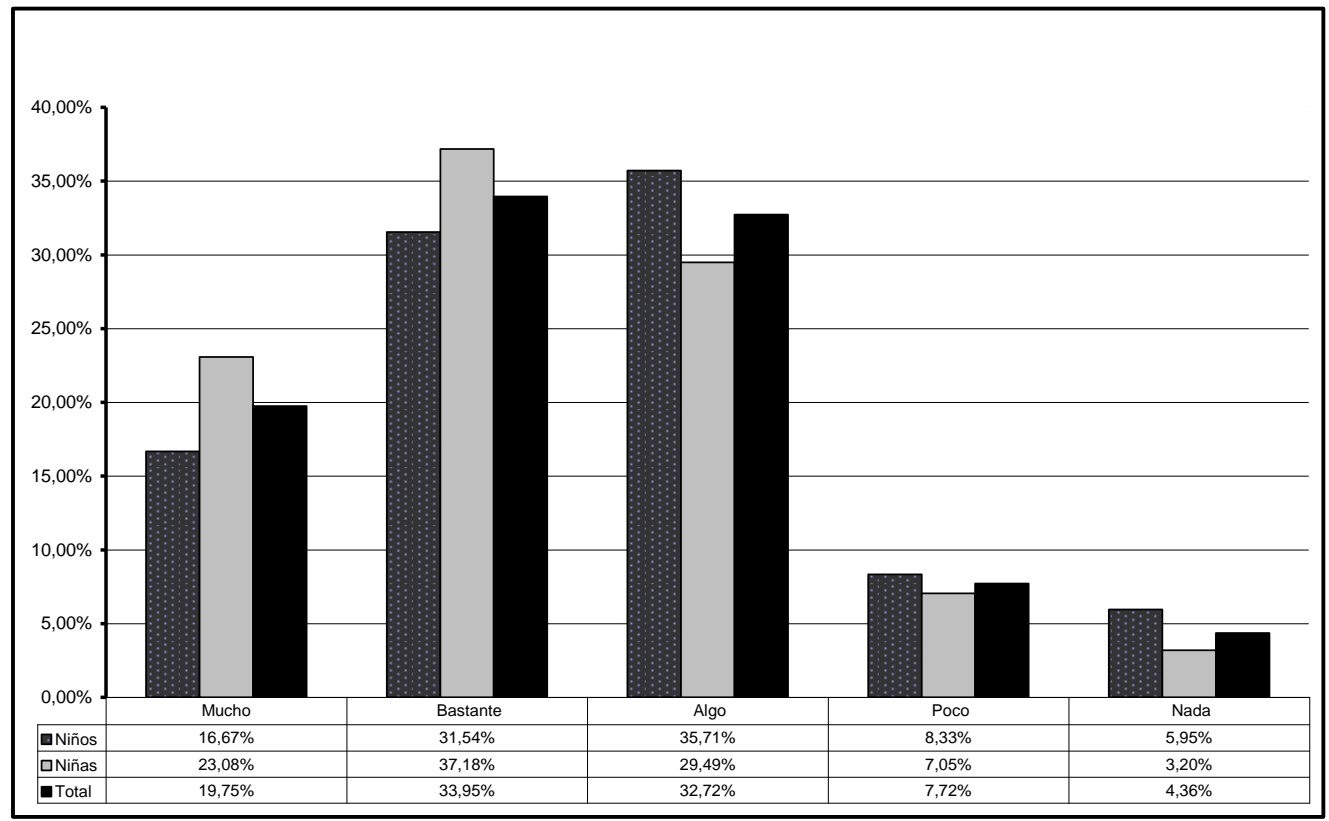

Figura 1. Valoración de la lectura: Les gusta leer. Fuente: elaboración propia.

Además, existen ciertas diferencias entre niños y niñas: Éstas manifiestan una actitud más favorable hacia la lectura, que gusta "mucho" o "bastante" al 62,26\% de las mismas, mientras que este porcentaje, en los niños, se queda en un $48,21 \%$.

Por otra parte, se han observado variaciones según el centro educativo. Mientras en algunos colegios el porcentaje de estudiantes a los que la lectura les gusta mucho o bastante ronda o supera el $60 \%$, en otros desciende hasta el $40 \%$ o incluso, en un caso, hasta el $34,78 \%$. En todos los casos se aprecia cierta correspondencia entre la 
actitud hacia la lectura y el nivel socio-económico medio de las familias del alumnado. Los grupos que se encuentran por encima de la media en cuanto a gusto por la lectura proceden de barrios con un contexto socio-económico medio o medio-alto, con mayor presencia de padres y madres con estudios superiores y, en algunos casos, dedicados a la docencia. De modo inverso, los centros donde es menor el número de escolares que dice disfrutar mucho o bastante de la lectura se corresponden con contextos de nivel socio-económico y cultural más bajo. En estos grupos aumenta sobre todo el porcentaje de personas que dicen disfrutar "algo" con esta actividad. Además, en los situados en los dos últimos puestos, asciende también notablemente (hasta porcentajes iguales o superiores al 20\%) el número de estudiantes a los que les gusta "poco" o "nada" leer.

\subsection{Razones para leer}

El principal motivo para leer (figura 2) es el disfrute, seguido del aprendizaje. En menor medida, otras razones para la lectura son: la necesidad de llenar el tiempo de ocio, el influjo de las familias y el consejo de los profesores. Todos estos motivos tienen una importancia similar en niños y niñas.

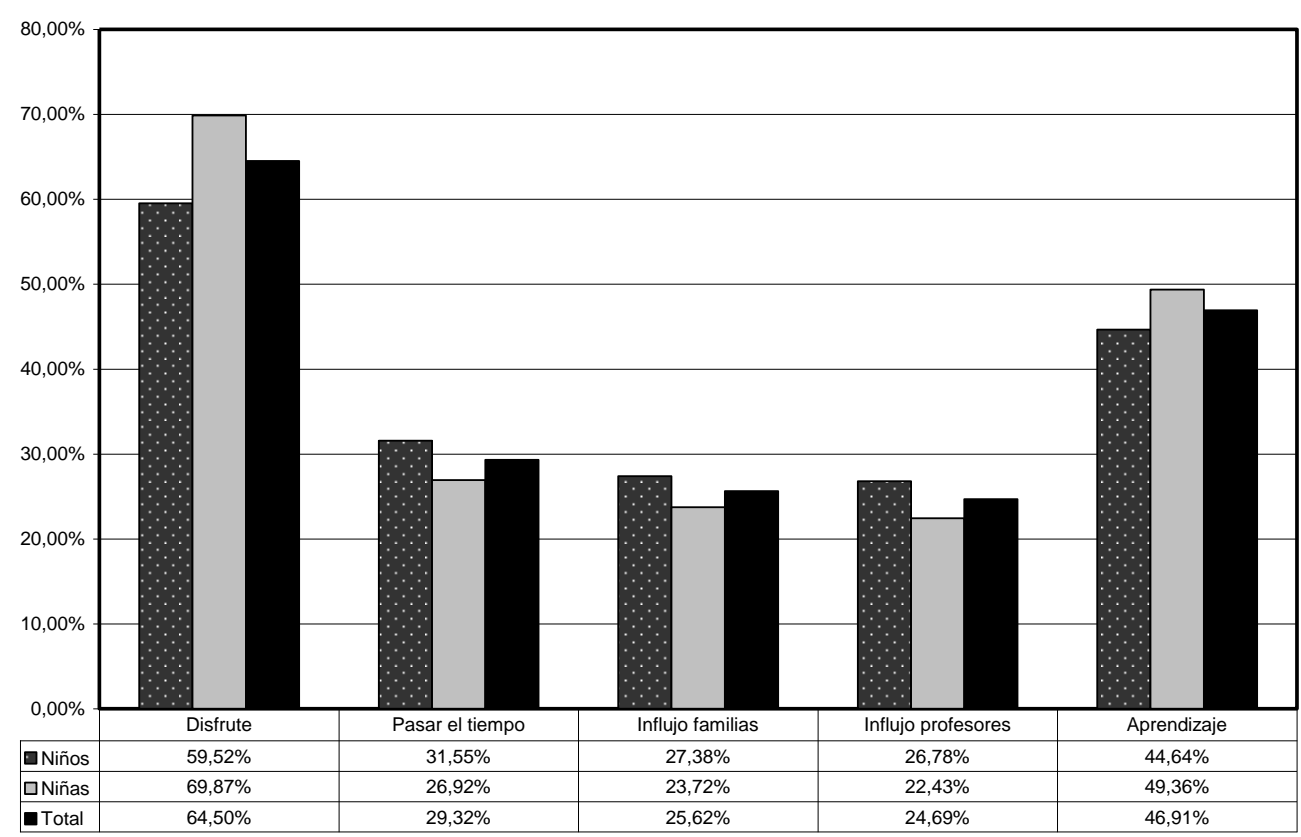

Figura 2. Razones para la lectura. Fuente: Elaboración propia.

El propósito de ocupar el tiempo de ocio con alguna actividad es citado por escolares a los que les gusta leer "algo" o "poco", mientras que raramente es señalado por parte de los niños y niñas a los que leer les gusta "mucho" o "bastante". Por otra 
parte, conviene prestar atención a la influencia que pueden ejercer los docentes y adultos de la familia en la decisión de leer, mientras que el influjo de los amigos apenas tiene repercusión, pues no alcanza en ningún caso el 5\%.

Por otra parte, en respuesta abierta los sujetos encuestados señalan otros motivos más específicos. La mayor parte de estas respuestas espontáneas se relacionan con el placer y entretenimiento producido por la lectura o con el disfrute de la literatura, vinculada sobre todo con su capacidad para crear mundos. Así, algunos escolares señalan simplemente que leer les gusta, entretiene o divierte. Otros apelan a la imaginación, la capacidad de vivir otras vidas, el interés de la intriga o la empatía con los personajes, con respuestas como: "me gusta imaginar que estoy dentro del libro", "me gusta entrar en las aventuras de los personajes", "siento que viajo a otro mundo", "es divertido leer ya que te lo imaginas como tú quieras", "es como si te pones en el lugar del personaje y sientes lo mismo que él", etc. Como vemos, estas respuestas remiten a la lectura literaria, haciendo énfasis en el placer de la ficción.

En menor medida, algunos expresan una valoración positiva de la lectura y su valor formativo, indicando que leer es "muy importante", "es fundamental para escribir bien", "es bueno", etc. Por último, un número muy reducido de escolares señala que lee porque en su casa les obligan.

\subsection{Causas del rechazo a la lectura}

Entre los escolares que muestran una actitud desfavorable a la lectura, la inmensa mayoría señala el aburrimiento que le produce esta actividad ("me aburro", "es un rollo", "me duermo", "no me divierte", etc.). Algunos citan otros motivos: les resulta cansado - tal vez debido a una baja competencia lectora-, no tienen tiempo para ello, o prefieren otras actividades de ocio como "el fútbol", "jugar" o "ver la tele". Otros matizan su rechazo indicando que sólo les gusta leer algunos libros o hacen referencia a la escasez de materiales disponibles que conecten con sus propios gustos. Por último, algunos no saben explicar las causas de su rechazo o proporcionan respuestas tautológicas ("porque no", "porque no me gusta", etc.) que no ofrecen ninguna justificación satisfactoria.

\subsection{La lectura como actividad de ocio}

$\mathrm{Al}$ ser preguntados en respuesta abierta por sus actividades de ocio preferidas, el $30,55 \%$ cita la lectura. Las actividades preferidas son, por este orden, practicar algún deporte -principalmente fútbol-, leer, utilizar el ordenador, jugar, ver la televisión, jugar con videojuegos, quedar con amigos y escuchar música. Hay otras actividades menos relevantes, no reflejadas en el gráfico por haber sido citadas en porcentajes inferiores al $10 \%$, como bailar (7,69\%), pasear o caminar $(5,86 \%)$, dibujar, pintar o hacer manualidades $(4,32 \%)$, estudiar $(3,39 \%)$, actividades familiares como visitar a los abuelos, ayudar en casa, acompañar a los padres (2,77\%), ocuparse de las mascotas $(1,85 \%)$, etc. 
Si consideramos los resultados procedentes de niños y niñas, observamos diferencias notables (figura 3). La lectura como actividad favorita de ocio es más citada por las niñas que por los niños, al igual que otras ocupaciones como jugar, ver la televisión, quedar con amigos y escuchar música.

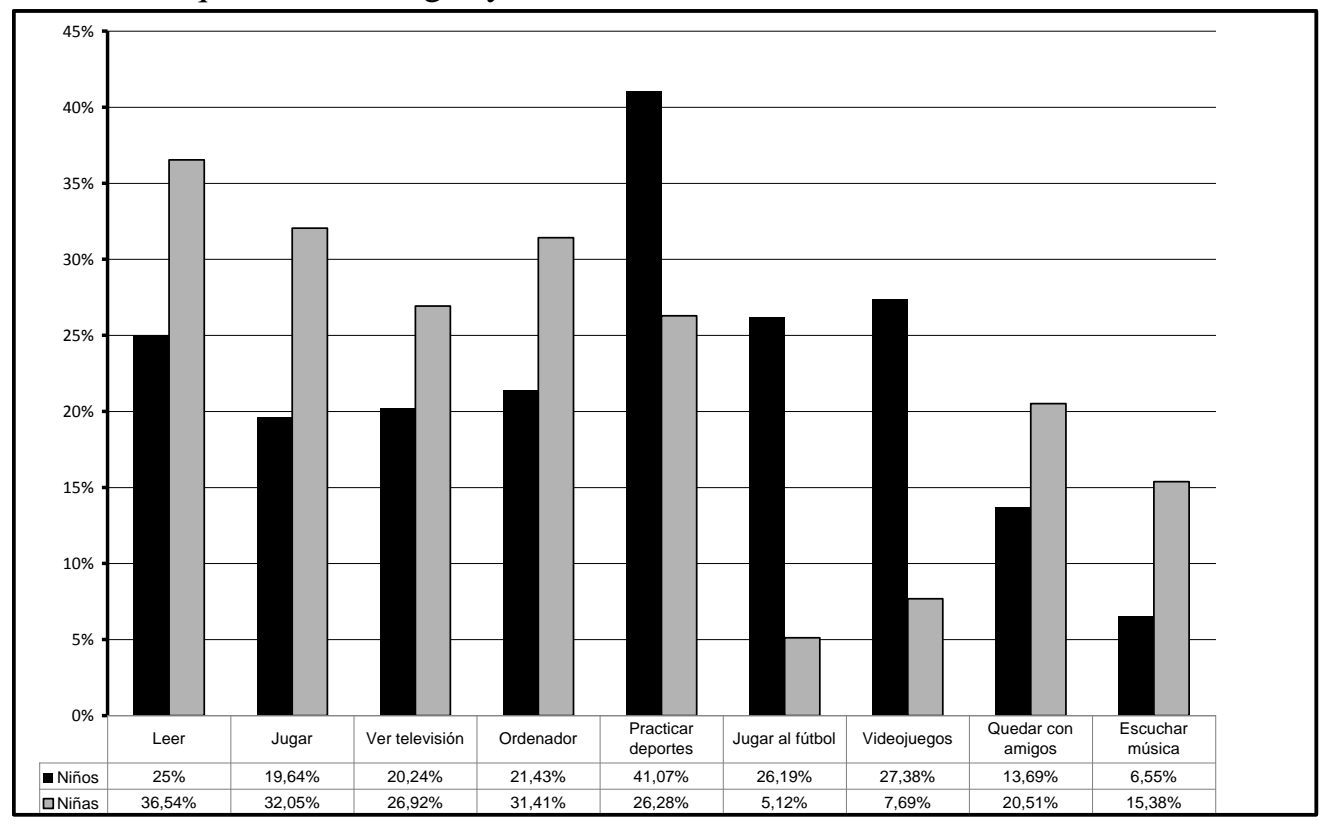

Figura 3. Actividades preferidas por niños y niñas Fuente: elaboración propia.

En cambio, la práctica de deportes -principalmente el fútbol- y los videojuegos son especial objeto de las preferencias de los niños. En términos generales, los escolares de sexo masculino manifiestan predilección por ocupaciones que implican actividad física, aunque también muestran su preferencia por los videojuegos, quedando la lectura en cuarta posición, por debajo de los deportes en general, los videojuegos y el fútbol. Entre las niñas, la lectura ocupa el primer lugar, seguida del juego, el ordenador y la televisión. La actividad física despierta también cierto interés, pero desciende a una quinta posición, muy por debajo del lugar que le otorgan los niños.

\subsection{Frecuencia de actividades de ocio}

La expresión de las preferencias sobre ocupaciones de ocio se ha completado con la obtención de datos sobre la frecuencia con que los escolares de ambos sexos practican diversas actividades fuera de la escuela. Ello nos ha permitido obtener una relación de actividades más habituales (diarias o frecuentes), y observar la posición que la lectura ocupa entre ellas (figura 4). 


\begin{tabular}{|l|l|l|l|}
\hline Niños & Niñas \\
\hline $\begin{array}{l}\text { Actividades } \\
\text { diarias }\end{array}$ & $\begin{array}{l}\text { Actividades fre- } \\
\text { cuentes (diarias o } \\
\text { semanales) }\end{array}$ & Actividades diarias & $\begin{array}{l}\text { Actividades fre- } \\
\text { cuentes (diarias o } \\
\text { semanales) }\end{array}$ \\
\hline $\begin{array}{l}\text { 1.Estudiar o } \\
\text { hacer deberes }\end{array}$ & 1.Ver televisión & $\begin{array}{l}\text { 1.Estudiar o hacer debe- } \\
\text { res }\end{array}$ & $\begin{array}{l}\text { 1.Estudiar o hacer } \\
\text { deberes }\end{array}$ \\
\hline $\begin{array}{l}\text { 2.Ver televisión } \\
\text { 2.Estudiar o hacer } \\
\text { deberes }\end{array}$ & 2.Ver televisión & 2.Ver televisión \\
\hline $\begin{array}{l}\text { 3.Practicar de- } \\
\text { porte }\end{array}$ & 3.Practicar deporte \\
música & 3. Practicar deporte & 3. Escuchar música \\
\hline $\begin{array}{l}\text { 5.Usar el orde- } \\
\text { nador }\end{array}$ & $\begin{array}{l}\text { 5.Jugar con video- } \\
\text { juegos }\end{array}$ & 5.Leer & 4.Practicar deportes \\
\hline $\begin{array}{l}\text { 6.Jugar a otros } \\
\text { juegos }\end{array}$ & 6.Escuchar música & 6.Usar el ordenador & 6.Leer \\
\hline $\begin{array}{l}\text { 7.Leer } \\
\text { 7. y 8. Jugar a otros } \\
\text { juegos/quedar con } \\
\text { amigos }\end{array}$ & 7.Jugar a otros juegos & $\begin{array}{l}\text { 7.Jugar a otros jue- } \\
\text { gos }\end{array}$ \\
\hline $\begin{array}{l}\text { 8.Jugar con } \\
\text { videojuegos }\end{array}$ & 9.Leer & 8.Jugar con videojuegos \\
\hline $\begin{array}{l}\text { 9. Quedar con } \\
\text { amigos }\end{array}$ & $\begin{array}{l}\text { 9. Q. Jugar con video- } \\
\text { juegos }\end{array}$ \\
\hline
\end{tabular}

Figura 4. Actividades de ocio de niños y niñas Fuente: elaboración propia.

La actividad más frecuente fuera de la escuela está vinculada, realmente, al ámbito escolar (estudiar o hacer deberes) -el 90\% la realiza diariamente. Excluyendo este tipo de tareas, los escolares de $6^{\circ}$ dedican su tiempo libre fundamentalmente a ver la televisión, practicar deportes, usar el ordenador, escuchar música, leer, jugar en general, quedar con amigos, y jugar con videojuegos, por este orden, de modo que la lectura se mantiene en un sexto lugar. Entre las actividades de frecuencia diaria, las más citadas son realizar deberes $(90 \%)$, ver la televisión $(71,30 \%)$, practicar deportes $(58 \%)$, escuchar música $(47,22 \%)$ y usar el ordenador $(38,27 \%)$, todas ellas situadas por encima de la lectura $(33,33 \%)$. Hay que tener en cuenta que algunas de estas actividades -estudiar o hacer deberes y utilizar el ordenador- exigen también leer, por lo que en realidad la frecuencia de lectura diaria fuera de la escuela es superior a la indicada explícitamente.

Niños y niñas realizan con frecuencia similar algunas actividades, como hacer deberes, utilizar el ordenador o ver la televisión, mientras que otras ocupaciones presentan variaciones más o menos significativas. Así, los niños dedican más 
tiempo a hacer deporte y a utilizar videojuegos, mientras que las niñas leen y escuchan música con más frecuencia que los niños.

\subsection{Frecuencia de lectura}

Si tenemos en cuenta la periodicidad con la que los encuestados dicen leer en su tiempo libre (figura 5), observamos que más de los dos tercios $(78,39 \%)$ son lectores frecuentes (leen diaria o semanalmente), aunque quienes declaran realizar esta actividad a diario se reducen a un $33,33 \%$. Un porcentaje muy bajo lee en su tiempo libre de forma solamente ocasional (al menos una vez cada tres meses). Idéntico porcentaje es el de quienes se declaran no lectores (no leen nunca o casi nunca). En total, los que dicen no leer o hacerlo de forma ocasional ascienden a un 12,96\%.

El porcentaje de escolares encuestados que dicen leer con frecuencia resulta inferior al número de adultos que se declaran lectores frecuentes -en cualquier tipo de material, formato o soporte- de acuerdo con investigaciones realizadas a nivel nacional, y que los últimos estudios de Conecta sitúan por encima del $80 \%$ (Conecta, 2012, 2013). Sin embargo, hay que hacer notar que estos datos relativos a la población adulta incluyen tanto la lectura en el tiempo de ocio como la ligada al trabajo, mientras que los resultados de nuestro trabajo se refieren únicamente a la lectura en el tiempo libre. Por otra parte, en este ítem del cuestionario se preguntaba por la lectura como actividad ocio en general, sin diferenciar entre lectura literaria y funcional, ni tampoco entre distintos formatos y soportes. Pese a ello, hemos observado que muchos escolares parecen identificar el concepto de lectura con el formato libro y con los materiales impresos, por lo que probablemente el número de lectores frecuentes es ligeramente superior a la cifra obtenida. De hecho, el número de los escolares de centros ovetenses que dicen leer frecuentemente es sólo ligeramente superior al de los que se declaran lectores frecuentes de libros.

Estos porcentajes presentan variaciones según el sexo, pues el 37,82\% de las niñas dice leer diariamente y un $86,54 \%$ lo hace con frecuencia (de modo diario o semanal). En cambio, el porcentaje de niños que dice leer diariamente desciende a un $29,17 \%$, mientras que el número de niños lectores frecuentes se sitúa en un $70,83 \%$. El número de niñas que no leen o leen poco también es más reducido que el de niños. Entre éstos, si sumamos los no lectores con los que practican esta actividad de forma ocasional (alguna vez al mes o alguna vez cada tres meses), el total asciende a un $17,25 \%$. 


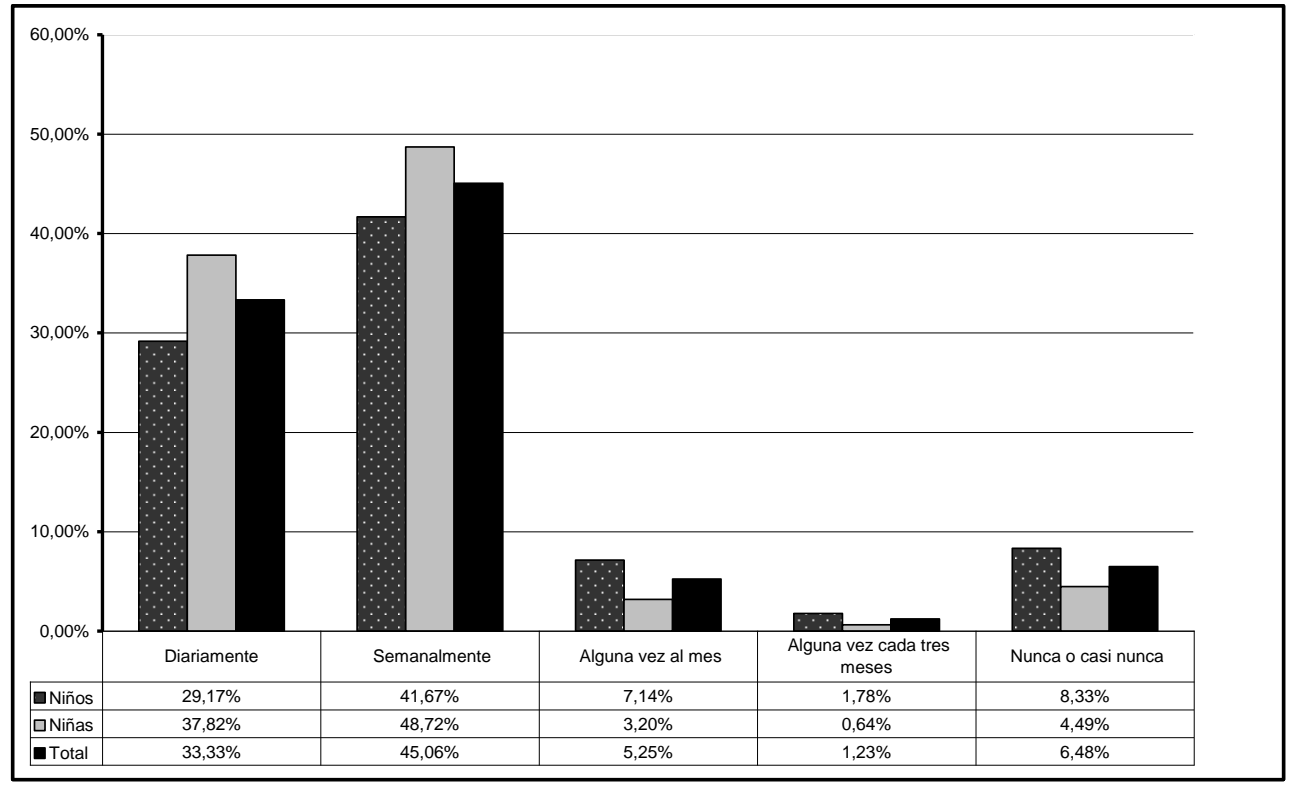

Figura 5. Frecuencia de lectura en el tiempo libre. Fuente: elaboración propia

Estos resultados generales presentan algunas variaciones según los centros educativos. En todos los colegios se mantiene la diferencia a favor de las niñas, que siempre superan a sus compañeros de sexo masculino en cuanto a lectura diaria. $\mathrm{Si}$ consideramos los lectores frecuentes, las niñas superan a los niños en casi todos los centros, o bien se mantienen prácticamente igualados.

En cambio, la frecuencia total de lectura varía significativamente según los colegios. En algunos centros los escolares que se declaran lectores frecuentes se sitúan entre el 80 y el $100 \%$, descendiendo en cambio en otros centros educativos hasta el $60,87 \%$. Lo mismo sucede con el porcentaje de lectores diarios, que oscila desde el $61,11 \%$ en uno de los colegios hasta el $17,39 \%$ en el otro extremo. Aunque existen ligeras variaciones según consideremos el número total de lectores frecuentes (diarios y semanales) o sólo el de lectores diarios, en términos generales, algunos colegios ocupan siempre posiciones altas, mientras que otros oscilan en niveles intermedios, y algunos pocos tienden a situarse siempre en los lugares más bajos de la escala. En todos los casos, las variaciones tienden a coincidir con variables socioeconómicas y confirman, en términos generales, los datos relativos a la valoración de la lectura ya comentados. En aquellos centros que se sitúan en posiciones más bajas, se observa también que la diferencia entre niñas y niños se acentúa a favor de las primeras, siendo los niños quienes hacen descender la frecuencia lectora. 


\subsection{Lectura en soportes digitales: uso del ordenador en el tiempo libre}

Además de practicar la lectura en papel, la mayoría lee con asiduidad en soportes digitales. Según se desprende de las respuestas obtenidas, los niños y niñas no consideran que usar el ordenador sea leer, dado que la frecuencia de lectura que declaran es inferior a la de uso de esta herramienta informática. Sin embargo, la utilización del ordenador en el tiempo de ocio, que alcanza porcentajes bastante elevados, implica al mismo tiempo tareas de lectura y escritura en soportes electrónicos, con el consiguiente desarrollo de habilidades comunicativas y digitales al mismo tiempo.

Fuera de la escuela, los estudiantes utilizan el ordenador para comunicarse, jugar y, en menor medida, para realizar tareas escolares. De hecho, parte de las actividades más citadas - chatear, realizar tareas de clase y buscar información- exigen leer y escribir en medios digitales. Si consideramos las variaciones entre niños y niñas, detectamos algunas diferencias en el uso que ambos realizan del ordenador. Más de la mitad de las niñas lo emplean principalmente para chatear. En menor medida, lo usan también, por este orden, para jugar, realizar deberes o tareas de clase, ver películas o escuchar música, buscar información, utilizar las redes sociales y -en un porcentaje ínfimo- para comunicarse mediante el correo electrónico. En cambio, los niños emplean el ordenador prioritariamente para jugar, y en menor medida para chatear, realizar tareas escolares, ver películas o escuchar música, buscar información, utilizar redes sociales y escribir o recibir e-mails.

\subsection{Frecuencia de lectura según tipos de textos}

En cuanto al tipo de lecturas en soporte impreso (figura 6), los escolares encuestados leen preferentemente libros, seguidos de revistas, cómics y periódicos. La mayoría de los estudiantes $(75,61 \%)$ es lector frecuente de libros, mientras que un cuarto lee libros de forma ocasional y un porcentaje reducido $(6,48 \%)$ dice no leer este tipo de materiales impresos nunca o casi nunca. Un elevado número de escolares se interesa también por las revistas, de las que casi la mitad es lector frecuente, mientras que casi un tercio las lee de forma ocasional. Algo menos de la mitad dice leer cómics con frecuencia, mientras que otro tanto los lee ocasionalmente. Por último, se observa gran diversidad en cuanto a la lectura de la prensa, pues los sujetos encuestados se reparten entre quienes dicen leerla de modo diario o semanal y quienes no la leen nunca o casi nunca.

En algunas encuestas, la frecuencia lectora de cómics, periódicos y revistas supera a la que los mismos sujetos han declarado en relación con la lectura en general. Podemos considerar que dichos estudiantes identifican el concepto de "lectura" con la de libros en soporte papel, excluyendo la lectura en otros soportes y formatos. También se ha observado la existencia de algunos escolares con bajo interés por la lectura en general y poca frecuencia lectora de libros que, sin embargo, sí dicen leer habitualmente revistas y cómics, por lo que estos formatos parecen despertar cierto interés entre los lectores reticentes. 


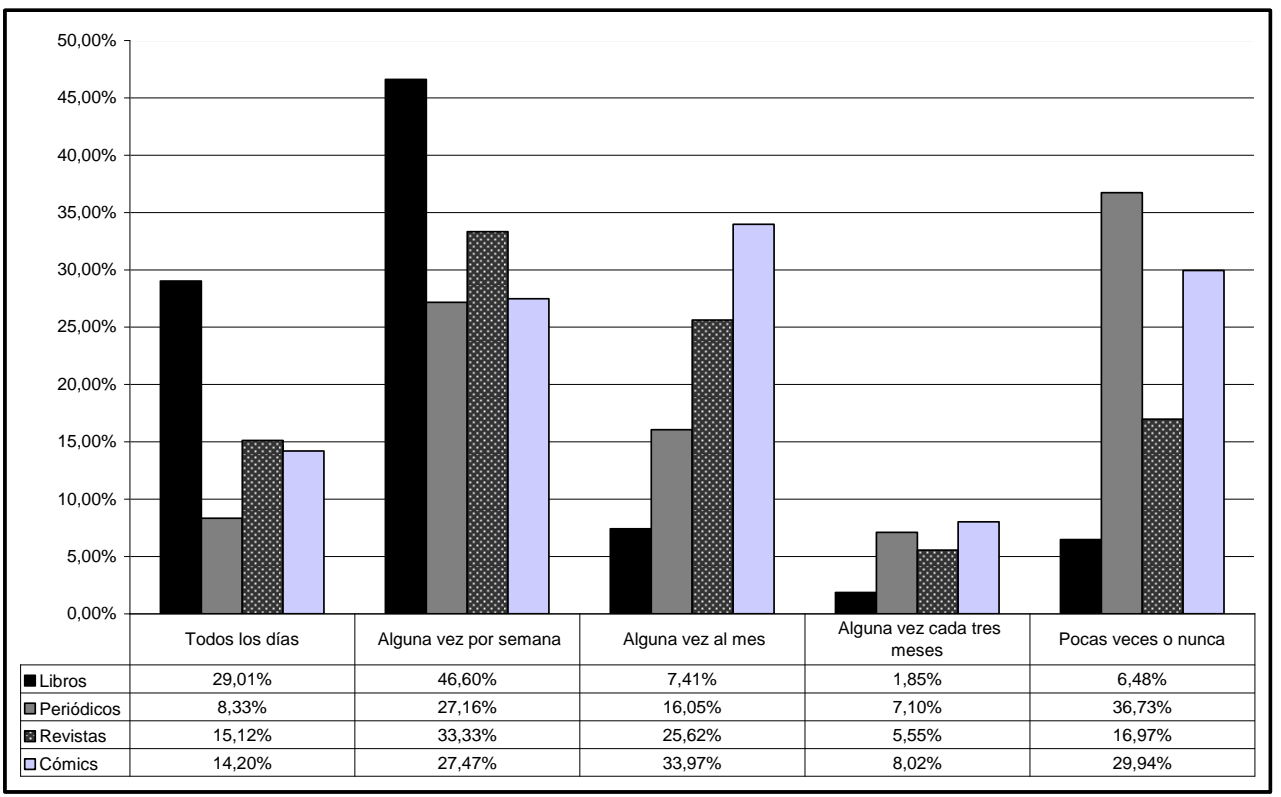

Figura 6. Frecuencia de lectura según tipos de textos. Fuente: elaboración propia.

Los porcentajes son bastante similares a los obtenidos por la encuesta realizada por Conecta ${ }^{6}$ en 2011 (Conecta, 2012), según la cual el 75,1 \% de los españoles menores de 14 años es lector frecuente de libros. Por otra parte, el número de lectores ocasionales de libros en los colegios ovetenses seleccionados supera al de la encuesta de Conecta de ese mismo año, mientras que el porcentaje de quienes dicen no leer nunca o casi nunca libros en notablemente inferior en nuestra muestra. De forma más general, el $84,87 \%$ de los escolares encuestados lee al menos una vez al trimestre en su tiempo libre, porcentaje ligeramente superior al total de niños que dicen leer en el tiempo de ocio (82,9\%) según la encuesta realizada en 2011 por Conecta.

Si comparamos estos resultados con los de la población adulta recogida en la citada encuesta de Conecta, observamos que los mayores de edad leen sobre todo periódicos $(77,6 \%)$, libros $(61,3 \%)$ y revistas $(46,3 \%)$, mientras que el número de lectores habituales de cómics es mucho más bajo (12,6\%). Otras encuestas realizadas confirman que la lectura de periódicos entre los menores aumenta con la edad mientras que desciende la de cómics (Gervais, 2001, 100-101) por lo que es posible que la lectura asidua de prensa en este momento esté apuntando tendencias que se incrementarán en los años sucesivos.

${ }^{6}$ Datos referidos a menores españoles con edades comprendidas entre los 10 y los 13 años. 
Considerando los resultados por sexos se aprecian diferencias significativas en la frecuencia de lectura de los tipos de textos citados. En primer lugar, aunque los libros son el tipo de lectura que más practican niños y niñas, observamos que el número de lectoras frecuentes supera al de los lectores frecuentes de sexo masculino. Además, el porcentaje de quienes no leen libros es superior entre los niños.

Otra variación es la referida a la frecuencia lectora de prensa, cómics y revistas. Mientras las niñas superan claramente a los niños en cuanto a lectura habitual de revistas, el número de lectores frecuentes de periódicos y cómics es significativamente superior entre los niños. Asimismo, el número de quienes no leen prensa y cómic aumenta de forma clara entre las niñas. Estas diferencias por sexos se observan igualmente en los datos obtenidos en las últimas encuestas de Conecta (Conecta, 2012, 2013), referidos a menores de 14 años.

Además, dado que estas tendencias coinciden con las que se dan en la población adulta (Conecta, 2011, 2012, 2013), las diferencias entre niños y niñas respecto a la lectura de cómics, revistas y prensa podrían deberse a la imitación de modelos adultos de su entorno más cercano, probablemente del ámbito familiar. La lectura frecuente de estos materiales puede relacionarse con el interés que suscitan determinadas temáticas (por ejemplo, en el caso de la prensa, su lectura podría relacionarse con el interés por noticias deportivas). El interés por unos u otros contenidos en las publicaciones periódicas y cómics también puede derivarse también de una imitación de los modelos adultos del entorno próximo, que proporcionan ejemplos de lo que la sociedad considera "lecturas femeninas" y "lecturas masculinas".

\section{CONCLUSIONES}

Los datos comentados nos permiten extraer algunas conclusiones sobre la importancia y lugar que ocupa la lectura como actividad de ocio entre los escolares asturianos de $6^{\circ}$ de Primaria, así como plantear algunas reflexiones y sugerencias en relación con la educación lectora:

- La lectura en el tiempo de ocio: La mayoría de los encuestados manifiesta una actitud bastante favorable hacia la lectura, que forma parte del tiempo de ocio de casi todos los niños y niñas de 11 y 12 años, incluso de aquellos que expresan sólo un interés más tibio por esta ocupación. Los escolares de $6^{\circ}$ de Primaria dedican su tiempo libre a ver la televisión, practicar deportes, usar el ordenador, escuchar música, leer, jugar en general, utilizar videojuegos y quedar con amigos, por este orden. Más de los tres cuartos de los escolares dice leer frecuentemente, aunque muchos de ellos parecen identificar el concepto de lectura con los textos impresos y el formato libro.

- Razones para leer: Los escolares dan prioridad al disfrute -ligado a la lectura literaria y al placer de la ficción- como principal motivo para la lectura, pero también conceden bastante importancia al aprendizaje. Ambas razones relacionadas, respectivamente, con la lectura literaria y con la lectura de libros de conocimientos- deben ser tenidas en cuenta por los docentes a la hora de se- 
leccionar y recomendar materiales para leer en el tiempo libre. Además, los escolares leen también por recomendación de profesores y adultos de la familia, por lo que es esencial prestar atención al influjo que los mediadores pueden ejercer en la formación y consolidación de hábitos lectores.

- No-lectores: Aunque el porcentaje de no lectores es bajo, hay que prestar atención a este grupo, que corre el riesgo de aumentar progresivamente durante la adolescencia y afianzar aún más su rechazo a la lectura. Un 12\% manifiesta un interés escaso o nulo hacia esta actividad debido al aburrimiento que le produce, y dice no leer nunca, o solamente de modo ocasional en su tiempo libre. Este porcentaje llega en los estudiantes de sexo masculino hasta un $17 \%$.

- Materiales de lectura y preponderancia del libro: Los escolares leen preferentemente libros, seguidos de revistas, cómics y periódicos, lo que coincide con los datos obtenidos sobre hábitos lectores de los menores a nivel nacional. Desde el punto de vista educativo, juzgamos importante tener en cuenta las preferencias manifestadas hacia los diferentes formatos, que no coinciden exactamente con las de los lectores adultos. Resulta claro que el gusto por la lectura y la formación del hábito lector en los menores se apoya fundamentalmente en la lectura de libros, aunque es importante tener en cuenta la presencia de otros tipos de textos.

- Cómics y revistas: Casi la mitad de los estudiantes lee habitualmente revistas y cómics, que podrían ser utilizados en el ámbito escolar como recursos para desarrollar la competencia lectora y animar a leer. Se ha visto cómo la percepción que algunos escolares tienen de sí mismos como lectores es errónea, por considerar que leer revistas o cómics no es leer. En este sentido, y a pesar del lugar fundamental que ocupa la lectura de libros en la formación de lectores competentes, es importante que se trabajen en el aula textos escritos pertenecientes a otros formatos, lo que contribuiría a cambiar el concepto que algunos escolares tienen de la lectura y, por consiguiente, su autopercepción como lectores. Además, la utilización de textos pertenecientes a cómics y revistas adecuadamente seleccionados por el docente podría constituir una vía de acercamiento a la lectura para aquellos estudiantes más reacios a esta actividad.

- La prensa: Hay estudiantes, principalmente varones, que dicen leer periódicos habitualmente, aunque, al mismo tiempo, son también muchos los que no los leen nunca o casi nunca. Desde el punto de vista educativo, el docente ha de tener en cuenta no sólo los tipos de textos que los alumnos prefieren sino también los que generan más rechazo. En lo que a la prensa se refiere, consideramos importante buscar formas adecuadas para acercar a los estudiantes a este tipo de material, proporcionándoles orientaciones para familiarizarse con este tipo de lectura, probablemente más difícil y menos atractiva para niños y niñas pero no por ello menos importante para su formación como lectores competentes. 
- Comparación con resultados nacionales: Los datos obtenidos no distan mucho de los del informe de Conecta de 2011 referidos a la lectura de libros en los menores, aunque con resultados ligeramente superiores en nuestra muestra, en cuanto a frecuencia lectora. En relación con los hábitos lectores de la población española en general, el número de lectores frecuentes en $6^{\circ}$ es algo inferior, aunque hay que recordar que muchos menores identifican la lectura solamente con determinados formatos. Por otra parte, los sujetos encuestados superan a la población española en lectura de libros y cómics, presentan resultados parecidos en lectura de revistas y obtienen resultados muy inferiores en lectura de prensa, que es la modalidad de texto más leído por la población en general.

- Actividades de ocio: El estudio revela cuáles son las actividades de ocio preferidas de forma mayoritaria por los escolares de 11 y 12 años, lo que puede ser importante a la hora de seleccionar textos para trabajar la lectura en la escuela. La selección de materiales escritos, tanto literarios como de otro tipo, vinculados con áreas de interés para los escolares -como los deportes, la música, la televisión, etc.- puede ser una forma de despertar el interés por la lectura e incluso, en algún caso, podría constituir una vía para estimular a los lectores reticentes y no lectores. De hecho, en otros ítems de la encuesta, no analizados en este trabajo, se ha observado cómo algunos lectores reticentes sí muestran cierto interés por textos que traten temáticas relacionadas con sus actividades favoritas, como el fútbol o los videojuegos.

- La importancia del contexto en la conformación del hábito lector: Aunque se han observado tendencias análogas en todos los colegios, se han detectado diferencias en actitud hacia la lectura en general y frecuencia lectora, que parecen apuntar a una relación entre el nivel económico y cultural del entorno y la actitud de los escolares hacia la lectura. Ello parece confirmar el influjo del contexto familiar en el interés por la lectura como actividad de ocio. Aunque la responsabilidad en la formación de hábitos lectores no puede recaer únicamente en la escuela, parece indudable que en los contextos menos favorables es necesaria una toma de conciencia de los problemas y un esfuerzo suplementario de fomento de la lectura desde el centro, para intentar suplir las carencias derivadas del entorno familiar.

- Diferencias por sexos en actitud y frecuencia lectora: Por último, se revelan diferencias en los hábitos lectores según el sexo. En términos generales, las niñas superan a los niños tanto en su interés hacia la lectura como en la frecuencia lectora, resultados que parecen corresponder a una constante supranacional, pues se repiten tanto en las encuestas realizadas en España (Conecta, 2012; Muñoz y Hernández, 2009) como en investigaciones análogas desarrolladas en otros países (Clark y Akerman, 2008, 2-3) y coinciden con tendencias similares en la población adulta. 
- Diferencias por sexos en preferencias lectoras: Niños y niñas difieren también en algunas de sus preferencias lectoras. Aunque ambos coinciden en su predilección por los libros como material de lectura, muestran divergencias en lo referido a otros tipos de textos. Mientras que las niñas leen más revistas, los niños, leen más cómics y más prensa. Dada la coincidencia con resultados obtenidos entre la población española adulta, ello se puede relacionar con divergencias en los gustos de ambos sexos, pero también con la imitación de modelos lectores proporcionados por la sociedad y por el entorno próximo.

\section{PROSPECTIVA}

En la investigación en la que se enmarca este trabajo se ha recabado información sobre otros aspectos que no se han tratado aquí, como el uso de bibliotecas, la selección de lecturas, las preferencias lectoras, así como una consideración más específica de la lectura literaria frente a la lectura ligada al aprendizaje. Estas cuestiones serán objeto de análisis en futuros trabajos, y permitirán ampliar el análisis y reflexión sobre los hábitos lectores de los escolares asturianos, completando los resultados expuestos en este trabajo.

Por otra parte, nos interesa profundizar en el análisis de los datos relativos a los lectores reticentes y no-lectores. Un análisis independiente de los resultados obtenidos en estos sujetos nos permitirá indagar en los diversos factores que explican su actitud negativa hacia la lectura.

Por último, dado que la localidad elegida para la aplicación de este estudio es representativa de un espacio urbano en el marco del Principado de Asturias, consideramos que los resultados obtenidos ofrecen elementos para la comparación con los hábitos lectores de escolares de Primaria de otras comunidades autónomas españolas.

\section{BIBLIOGRAFÍA}

CERrillo, Pedro y GARcía PADRINO, Jaime (Coords.) (1996): Hábitos lectores y animación a la lectura, Cuenca, Universidad de Castilla-la-Mancha.

CERrillo, Pedro; LARrAÑAgA, Elisa y YuBERo, Santiago (2002): Libros, lectores $y$ mediadores, Cuenca, Universidad de Castilla-la-Mancha.

CERRILlo, Pedro (2007): Literatura infantil y juvenil y educación literaria, Barcelona, Octaedro.

ClARK, Christina y AKERMAN, Rodie (2008): Being a Reader. The relationship with gender, Londres, National Literacy Trust.

http://www.literacytrust.org.uk/research/nlt_research/267_being_a_reader_the_r elationship_with_gender [Consulta: 7 de enero de 2013]

Clark, Christina y HaWkins, Lucy (2010): Young People's Reading: The Importance of the home environment and family support, Londres, National Literacy Trust. 
http://www.literacytrust.org.uk/research/nlt_research/2055_young_people_s_rea ding_the_importance_of_the_home_environment_and_family_support [Consulta: 7 de enero de 2013]

Clark, Christina; WoOdLeY, Jane y LeWIS, Fiona (2011): The Gift of Reading in 2011. Children and young people's access to books and attitudes towards reading, Londres, National Literacy Trust.

http://www.literacytrust.org.uk/research/nlt_research/4166_the_gift_of_reading _ in_2011_children_and_young_peoples_access_to_books_and_attitudes_towards _reading [Consulta: 7 de enero de 2013]

COLOMER, Teresa (Coord.) (2009): Lecturas adolescentes, Barcelona, Graó.

CONECTA (2011): Estudio de segmentación de lectores en España 2010: análisis multivariable. Madrid, Federación de Gremios de Editores de España/MEC.

CONECTA (2012): Hábitos de lectura y compra de libros en España 2011. Madrid, Federación de Gremios de Editores de España/Ministerio de Cultura.

CONECTA (2013): Hábitos de lectura y compra de libros en España 2012. Madrid, Federación de Gremios de Editores de España/Ministerio de Cultura.

DUEÑAS LORENTE, José Domingo (2013): "La educación literaria. Revisión teórica y perspectivas de futuro", en Didáctica. Lengua y Literatura, 25, 135-156.

GASOL, Anna y ARÀNEGA, Mercè (2000): Descubrir el placer de la lectura: lectura y motivación lectora, Barcelona, Edebé.

GERVAIS, Flore (2001): "Habitudes de lecture chez les 9-12 ans: une enquête aux résultats qui nous laissent songeurs", en Canadian Children's Literature, 100/101, 94-107.

GIL FLORES, Javier (2009): "Hábitos y actitudes de las familias hacia la lectura y competencias básicas del alumnado", en Revista de Educación, 350, 301-302.

GóMEZ SOTO, Ignacio (1999): Mito y realidad de la lectura. Los hábitos lectores en la España actual, Madrid, Endymion.

LARRAÑAGA, Elisa y YUBERO, Santiago (2005): "El hábito lector como actitud. El origen de la categoría de 'falsos lectores"', en Ocnos, 1, 43-60.

LINNAKYLÄ, Pirjo y VÄLIJÄRVI, Jouni (2006): "Rendimiento de los estudiantes finlandeses en PISA. Las claves del éxito en lectura", en Revista de Educación, número extraordinario 2006, 227-235.

LEY ORGÁNICA 2/2006, de 3 de mayo, de Educación. BOE nº106, Madrid, Ministerio de Educación y Ciencia.

MANRESA POTRONY, Mireia (2009): "Lecturas juveniles: el hábito lector dentro y fuera de las aulas", en Textos de Didáctica de la Lengua y de la Literatura, 51, 44-54.

MATA, Juan (2011): 10 ideas clave. Animación a la lectura, Barcelona, Graó.

MiLLÁN, José Antonio (coord.) (2002): La lectura en España. Informe 2002, Madrid, Federación de Gremios de Editores.

MORENO BAYONA, Víctor (2005): "Lectores competentes", en Revista de Educación, número extraordinario 2005, 153-167. 
MoRENO SÁNCHEZ, Emilia (2001): "Análisis de la influencia de la familia en los hábitos lectores de sus hijas e hijos: un estudio etnográfico", en Contextos educativos, 4, 177-179.

MuÑOZ RodRíGUEZ, José Manuel y HERNÁNDEZ MARTín, Azucena (2009): “Hábitos lectores de los alumnos de la ESO en la provincia de Salamanca. ¿Son el género y el entorno factores diferenciales?", en Revista de Educación, 354, 605628.

MURILlo TORRECILlA, Francisco Javier, et al. (2003): Los hábitos lectores de los adolescentes españoles, Madrid, MECD, CIDE.

OECD (2012): Informe PISA 2009: Aprendiendo a aprender: Implicación, estrategias y prácticas de los estudiantes (Volumen III). Santillana.

REYNOLDS, Kimberly. (2005): “¿Qué leen los jóvenes? Una comparación de los hábitos lectores en Australia, Dinamarca, Inglaterra e Irlanda", en Ocnos, 1, 87107.

VALDÉS, Mónica. (2013): “¿Leen en forma voluntaria y recreativa los niños que logran un buen nivel de Comprensión Lectora?", en Ocnos, 10, 71-89. 